54, 2, pp. 613-619, Warsaw 2016

DOI: $10.15632 /$ jtam-pl.54.2.613

\title{
ON THE BUCKLING AND VIBRATIONAL RESPONSE OF CARBON NANOTUBES WITH SPIRAL DEFORMATION
}

\author{
Sadegh Imani YengeJeh \\ Department of Solid Mechanics and Design, Faculty of Mechanical Engineering, Universiti Teknologi Malaysia - UTM, \\ Skudai, Johor, Malaysia; e-mail: imani.sd@gmail.com
}

Seyedeh Alieh Kazemi

Department of Mechanical Engineering, The University of Birjand, Birjand, Iran; e-mail: sa.kazemi83@gmail.com ANDREAS ÖCHSNER

School of Engineering, Griffith University, Gold Coast Campus, Southport, Australia, and

School of Engineering, The University of Newcastle, Callaghan New South Wales, Australia

e-mail: andreas.oechsner@gmail.com

\begin{abstract}
Perfect and spiral models of carbon nanotubes (CNTs) have been simulated based on the finite element method and their vibrational and buckling behavior has been investigated. In order to evaluate their natural frequency and critical buckling load, computational tests have been conducted. It has been concluded that the existence of any geometrical modification in the configuration of perfect CNTs results in a remarkable reduction in the natural frequency and critical buckling load of CNTs. It has been also revealed that the analytical solutions are in good agreement with the finite element simulation results in the cases of perfect and spiral CNTs.
\end{abstract}

Keywords: finite element method, carbon nanotubes, natural frequency, critical buckling load, spiral shape

\section{Introduction}

The industrial application of carbon nanotubes (CNTs) is continuously increasing due to their outstanding physical properties. Since their discovery (Iijima, 1991), these nanostructures have attracted worldwide attention. CNTs are unique because of their outstanding mechanical and physical properties such as strength, lightness and good conductivity (Dai et al., 1996; Saito et al., 1997; Niu et al., 1997). The investigation of CNTs can be divided into two groups, i.e. experimental and computational approaches. Molecular dynamics (MD) and continuum mechanics techniques such as the finite element method (FEM) have been the most popular approaches to study the mechanical behavior of these nano-materials. In the following, the results of several studies on vibrational characteristics and buckling behavior of CNTs are presented.

Previous investigations (Arghavan and Singh, 2011) conducted a numerical study on free and forced vibrations of single-walled carbon nanotubes (SWCNTs). They applied a simple approach so that the proximity of the mathematical model to the actual atomic structure of the CNT was considerably retained. Their results revealed that the appearance of these modes of vibration in the eigenvectors and eigenvalues were indistinguishable. It was concluded that in the case of zigzag nanotubes, the axial bending and torsional modes appeared to be decoupled whereas the armchair nanotubes showed coupling between such modes. Then, the vibrational behavior of twoand three-junctioned CNTs was investigated with different geometries and boundary conditions (Seyyed Fakhrabadi et al., 2012). The authors applied a molecular mechanics approach to analyze 
the mentioned CNTs. They finally obtained natural frequencies and their corresponding mode shapes of two-junctioned CNTs with different diameters and lengths. After that, the natural frequency of CNTs was investigated (Ghavamian and Öchsner, 2013). They simulated numerous forms of CNTs from single to 5-walled ones in their perfect form based on the FEM. Then, they evaluated natural frequencies of the CNTs analytically and through the FEM and compared their findings. Then, some specific defects were introduced to the perfect models and their vibrational behavior and the influence of these defects on the vibrational stability of CNTs were studied. They finally concluded that the existence and development of any type of defects in the configuration of CNTs reduce the natural frequency and vibrational stability of perfect CNTs. A study on the impact of vacancy defects on critical buckling loads and strains in CNTs was conducted under axial compression (Parvaneh et al., 2009). Their results showed that vacancy defects in CNTs can most likely be modeled as cutouts of the shells. They finally compared their results of the structural model with those from MD simulations in which the outputs were in good agreement with the present model. The buckling characteristics of several curved forms of SWCNTs was investigated performing MD simulations (Wong and Vijayaraghavan, 2012). They concluded that the performance of the CNT under compression can be changed by an inclusion of a curvature along the tube axis. The effect of defects on the buckling behavior of CNTs was investigated by Ghavamian and Öchsner (2012). Their study was based on the FEM. In detail, they modeled two basic CNTs in their perfect form. Then the buckling behavior of CNTs was evaluated by comparing their critical loads obtained from the simulation and analytical calculations. They concluded that the existence of any curvature in the structure of nanotubes decreases their buckling strength. The aim of the actual research is to continue the previous investigations and to study vibrational and buckling behavior of spiral CNTs.

As most of the investigations have been performed on perfect CNTs, it is necessary to pay more attention to geometrical imperfections, e.g. twists and spirals of CNTs, in order to realistically examine their mechanical properties (see Fig. 1). Spiral deformations were reported in (Faria et al., 2013; Liuyue et al., 2013; Faria et al., 2013) where the CNTs revealed a shape similar to a spring which is deformed around its longitudinal axis. The purpose of this research is to derive computational models of CNTs and investigate their vibrational and buckling behavior.

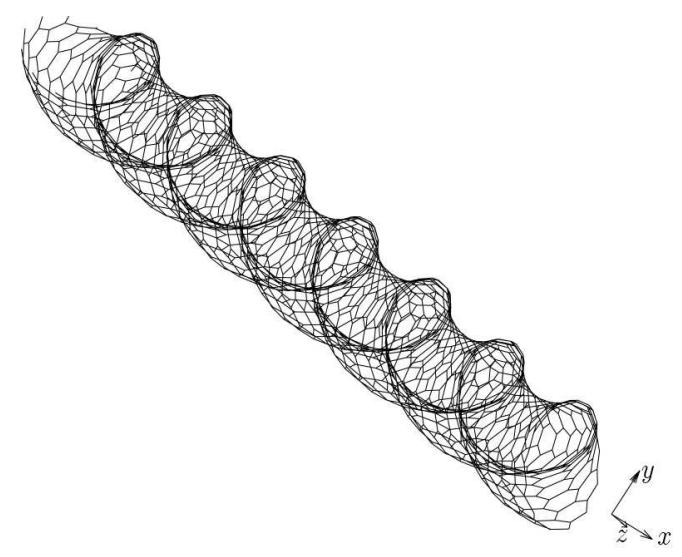

Fig. 1. General view of a spiral CNT

\section{Methodology}

\subsection{Geometric definition}

The atomic structure of CNTs can be imagined as a graphene sheet that has been rolled into a tube. The thickness of the tube wall is generally considered to be $0.34 \mathrm{~nm}$, which is very 
close to that of a graphene sheet ( $\mathrm{Li}$ and Chou, 2003; To, 2006). These nano-particles possess a length of over $10 \mu \mathrm{m}$ and diameters ranging from 0.4 to $3.0 \mathrm{~nm}$.

We followed the modeling method which was first proposed by Li and Chou (2003) where the theory of classical structural mechanics was extended to the modeling of CNTs. It was assumed that CNTs, when subjected to loading, behave like space-frame structures. Based on Fig. 2, the bonds between carbon atoms are considered as connecting load-carrying generalized beam members, while the carbon atoms act as joints of the members.

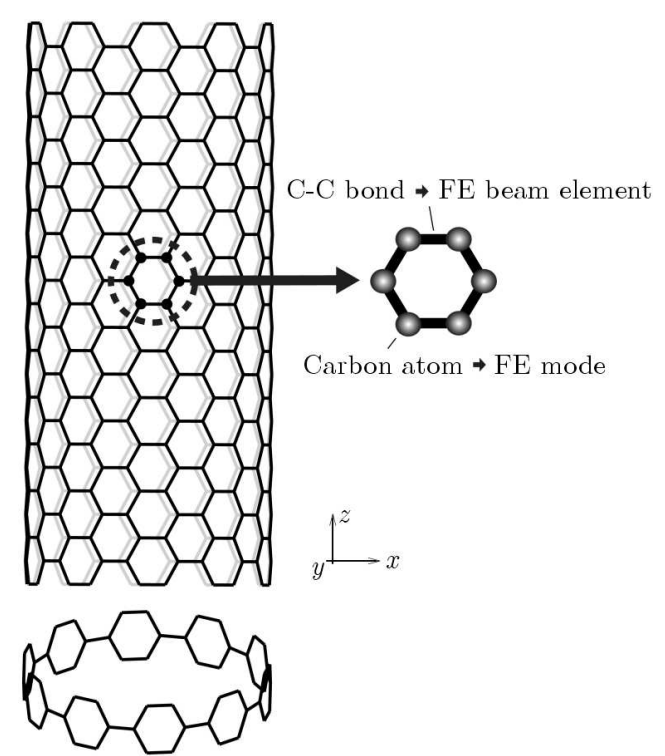

Fig. 2. Front view and single ring of a SWCNT as a space-frame structure

The configuration of CNTs in this study has been generated by the CoNTub software (Melchor and Dobado, 2004; Melchor et al., 2011), a computer program for determining the coordinates of CNTs. Then, the finite element analyses have been conducted in order to investigate the vibrational and buckling behavior of different types of perfect and spiral CNTs.

\subsection{Boundary conditions}

Vibrational and bucking behavior of perfect and spiral CNTs under cantilevered boundary conditions are investigated, where for the vibrational behavior one end is fully fixed and the other end is completely free; and for the buckling behavior one end is fully fixed and the other end is exposed to a compressive axial load. Different angles for spiral CNTs have been taken into consideration, as shown in Fig. 3.

\section{Results and discussion}

In order to evaluate the critical buckling load of CNTs, the numerical finite element approach allows the introduction of arbitrary compressive point loads to one of the CNT's ends. The simulation of the vibrational behavior does not require the introduction of any loads. The obtained numerical finite element results are compared to simple analytical predictions to check if these simple design equations provide reasonable values.

\subsection{Analytical approach to the vibrational behavior}

The natural frequency is the frequency of a vibrating system at which the system oscillates at a greater amplitude. This phenomenon occurs because of the existence of resonance. The natural 

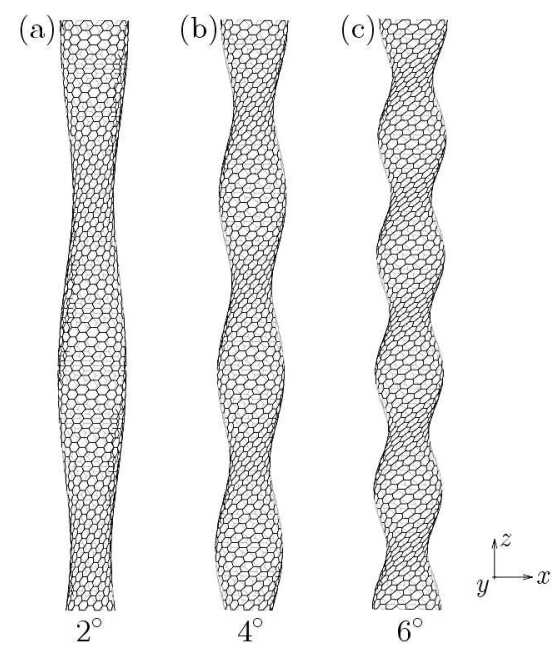

Fig. 3. Spiral CNT with (a) $2^{\circ}$, (b) $42^{\circ}$ and (c) $62^{\circ}$ twisting angle

frequency is mostly investigated to examine the vibrational response of structural members. The first natural frequency of a fixed-free helical spring is defined by the following analytical equation (Renno and Mace, 2012)

$$
f_{n}=\frac{1}{4} \sqrt{\frac{k}{\bar{m}}}
$$

where $\bar{m}$ and $k$ are the value of mass per unit of the spring and the spring rate, respectively. In the case of spiral CNTs, we assumed an average value for the diameter as the whole structure does not possess a constant diameter along its length. The value of the mass per unit length $\bar{m}$ can be obtained from the following equation

$$
\bar{m}=\frac{m_{\text {total }}}{l_{\text {total }}}
$$

where $m_{\text {total }}$ is the total mass of the CNT carbon atoms and $l_{\text {total }}$ is the total length of the nanotube. Figure 4 illustrates the first five natural frequencies of a spiral CNT obtained from the numerical finite element simulation.

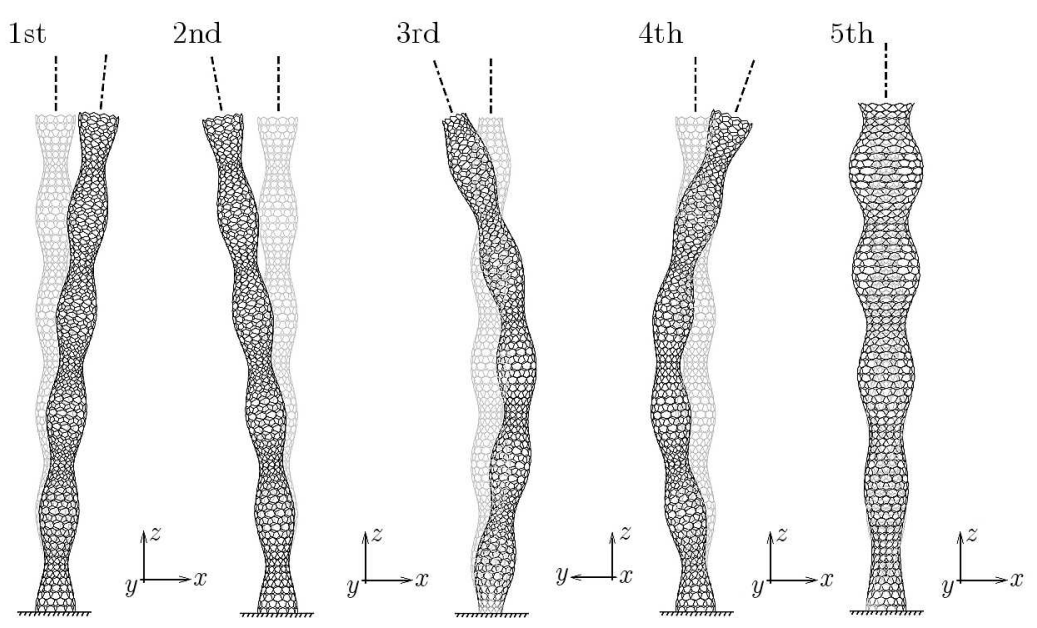

Fig. 4. First five eigenmodes of a spiral CNT 


\subsection{Analytical approach to the buckling behavior}

The phenomenon of buckling is in its simplest form a particular kind of elastic instability in a slender configuration that occurs under certain compressive loads. In the basic theory of elasticity, the critical buckling deflection of a compressive spring is presented by Eq. (3.3) (Pearson, 1982) as

$$
\frac{\delta_{c r}}{L_{f}}=0.812\left[1 \pm \sqrt{1-6.87\left(\frac{2 D_{m}}{L_{f}}\right)^{2}}\right]
$$

where $L_{f}$ is the free length or unloaded length of the spring, $D_{m}$ is the mean diameter of the spring, and $\delta_{c r}$ is the critical deflection of the spring.

Figure 5 shows a spiral CNT under the cantilevered boundary condition in its original and buckled shape obtained from the numerical finite element calculation.

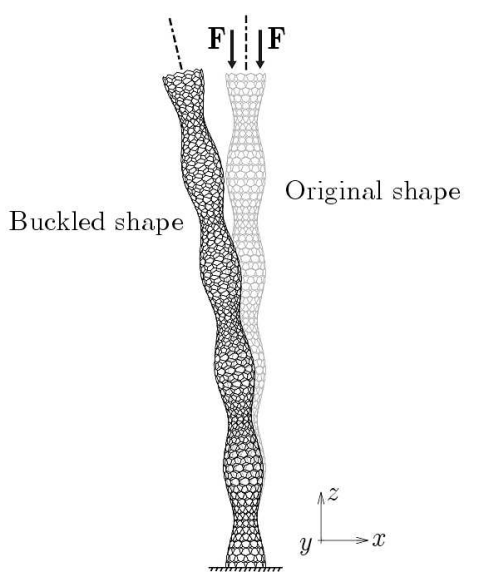

Fig. 5. A spiral CNT's first mode under buckling load in the original and buckled form with the cantilevered boundary condition

It should be indicated that the proposed approach in this paper deals with the evaluation of mechanical properties, i.e. vibrational and buckling behavior of spiral CNTs, whereby the CNTs are deformed as a spring around their axis. By manipulating the twisting angle of these spiral CNTs from $02^{\circ}$ to $102^{\circ}$, the behavior of different models with different conditions could be studied. This study tried to continue and broaden the investigations on only slightly twisted CNTs around their straight vertical axis.

It could be shown that the computational and analytical values of the natural frequency as well as critical buckling load of perfect CNTs are reduced by introducing spiral imperfections to the configuration of these perfect nano-structures, as illustrated in Fig. 6. Comparing all cases, it is clear that a decrease in the natural frequency and the critical buckling load is more visible in the case of armchair spiral CNTs. Figure 6a-c shows a decrease in the natural frequency of armchair, zigzag and chiral CNTs as a result of increasing the twisting angle of spiral configurations. It is clear that the natural frequency of all CNTs reduces to less than $12 \mathrm{GHz}$ at the angle of $102^{\circ}$. Figure $6 \mathrm{~d}$-f illustrates a significant change in the critical buckling load of armchair, zigzag and chiral models by increasing the twisting angle of spiral CNTs. Based on the obtained calculations, it is concluded that the finite element values are in good agreement with analytical results, where the maximum difference for the natural frequency and critical buckling load is 17 and $23 \%$, respectively. 
(a)

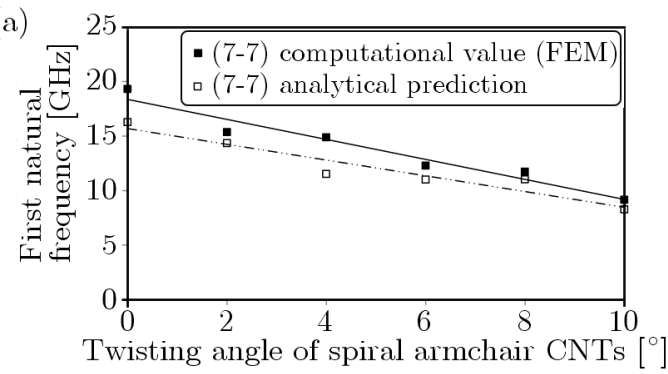

(c)

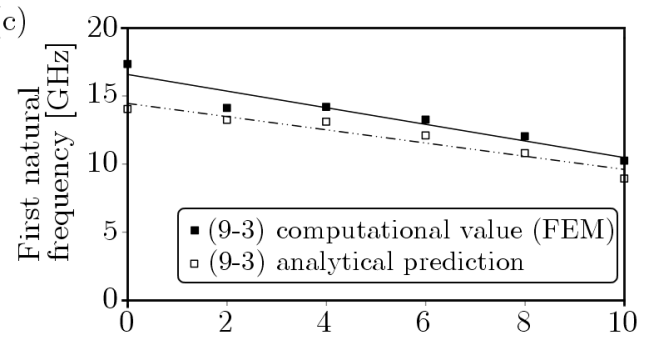

Twisting angle of spiral chiral CNTs $\left[^{\circ}\right.$

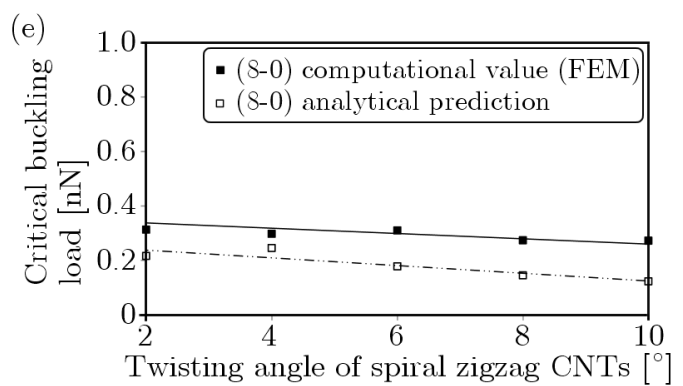

(b)

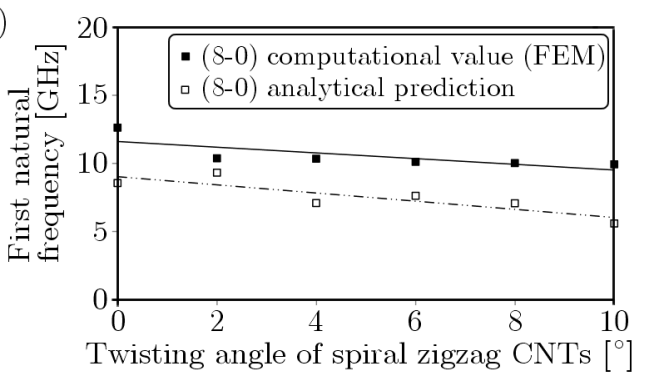

(d)

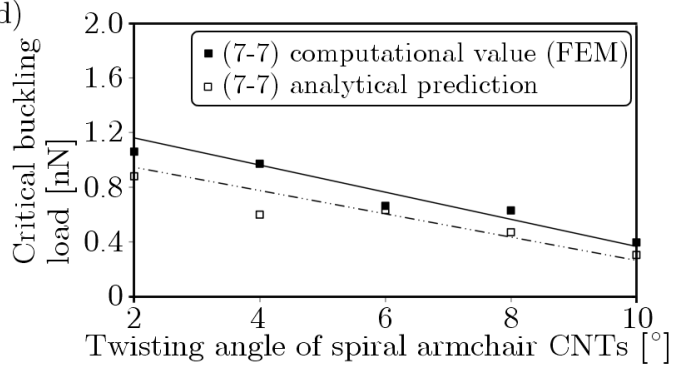

(f)

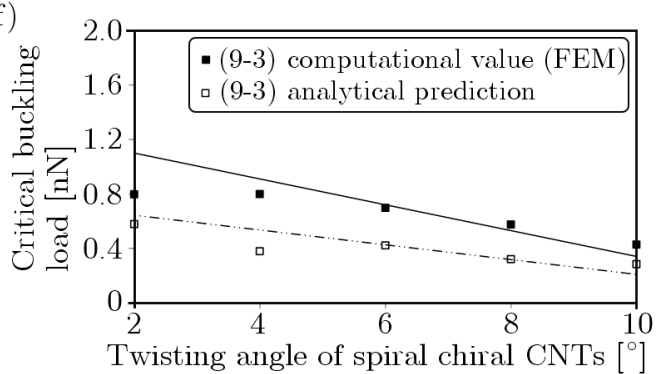

Fig. 6. Change in the natural frequency with different twisting angles for (a) armchair, (b) zigzag and

(c) chiral spiral CNTs. Change in the critical buckling load with different twisting angles for (d) armchair, (e) zigzag and (f) chiral spiral CNTs

\section{Conclusions}

In this study, perfect and spiral CNTs (armchair, zigzag and chiral) have been simulated and their vibrational and buckling behavior has been studied through performing computational tests with cantilevered boundary condition. Both computational and analytical calculations have been compared in the cases of perfect and spiral CNTs. It has been shown that the finite element simulation are in good agreement with the analytical solutions in the case of perfect and spiral CNTs. It has been shown that in all cases, the analytical values are slightly smaller than the computational results. It has been also concluded that the existence of any spiral imperfection in the configuration of perfect CNTs results in a significant reduction in the natural frequency and critical buckling load of these nano-configurations.

\section{References}

1. Arghavan S., Singh A.V., 2011, On the vibrations of single-walled carbon nanotubes, Journal of Sound and Vibration, 330, 3102-3122

2. Dai H., Wong E.W., Lieber C.M., 1996, Probing electrical transport in nanomaterials: conductivity of individual carbon nanotubes, Science, 272, 523-526

3. Faria B., Silvestre N., 2013, Canongia Lopes JN: Induced anisotropy of chiral carbon nanotubes under combined tension-twisting, Mechanics of Materials, 58, 97-109 
4. Faria B., Silvestre N., Canongia Lopes J.N., 2013, Tension-twisting dependent kinematics of chiral CNTs, Composites Science and Technology, 74, 211-220

5. Ghavamian A., ÖChsner A., 2012, Numerical investigation on the influence of defects on the buckling behavior of single-and multi-walled carbon nanotubes, Physica E, 46, 241-249

6. Ghavamian A., ÖChsner A., 2013, Numerical modeling of the eigenmodes and eigen frequencies of carbon nanotubes under the influence of defects, Journal of Nano Research, 21, 159-164

7. IıJima S., 1991, Helical microtubules of graphitic carbon, Nature, 354, 56-58

8. Li C., Chou T.W., 2003, A structural mechanics approach for the analysis of carbon nanotubes, International Journal of Solids and Structures, 40, 2487-2499

9. Liuyue C., Yang L., Hongbo L., Yuxi C., Xiaohong X., Haibo Z., 2013, Investigation of graphite/carbon spiral nanoribbons using $\mathrm{FeCl3}-\mathrm{CuCl}$-graphite intercalation compounds as precursors, Materials Letters, 108, 196-199

10. Melchor S., Dobado J.A., 2004, CoNTub: An algorithm for connecting two arbitrary carbon nanotubes, Journal of Chemical Information and Computer Sciences, 44, 1639-1646

11. Melchor S., Martin-Martinez F.J., Dobado J.A., 2011, CoNTub v2. 0-algorithms for constructing c 3-symmetric models of three-nanotube junctions, Journal of Chemical Information and Modeling, 51, 1492-1505

12. Niu C., Sichel E.K., Hoch R., 1997, High power electrochemical capacitors based on carbon nanotube electrodes, Applied Physics Letters, 70, 1480-1482

13. Parvaneh V., Shariati M., MajdSabeti A.M., 2009, Investigation of vacancy defects effects on the buckling behavior of SWCNTs via a structural mechanics approach, European Journal of Mechanics A/Solids, 28, 1072-1078

14. Pearson D., 1982, The transfer matrix method for the vibration of compressed helical springs, Journal of Mechanical Engineering Science, 24, 163-171

15. Rahmandoust M., ÖChsner A., 2011, Buckling behavior and natural frequency of zigzag and armchair single-walled carbon nanotubes, Journal of Nano Research, 16, 153-160

16. Renno J.M., Mace B.R., 2012, Vibration modelling of helical springs with non-uniform ends, Journal of Sound and Vibration, 331, 2809-2823

17. Saito Y., Hamaguchi K., Hata K., 1997, Conical beams from open nanotubes, Nature, 389, 554-555

18. Seyyed Fakhrabadi M.M., Amini A., Rastgoo A., 2012, Vibrational properties of two and three junctioned carbon nanotubes, Computational Materials Science, 65, 411-425

19. To C.W.S., 2006, Bending and shear moduli of single-walled carbon nanotubes, Finite Elements in Analysis and Design, 42, 404-413

20. Wong C.H., Vijayaraghavan V., 2012, Nanomechanics of imperfectly straight single walled carbon nanotubes under axial compression by using molecular dynamics simulation, Computational Materials Science, 53, 268-277 